\title{
Camera-Projector Calibration Methods with Compensation of Geometric Distortions in Fringe Projection Profilometry: A Comparative Study
}

\section{Métodos de calibración cámara-proyector con compensación de distorsiones geométricas en perfilometría por proyección de franjas: un estudio comparativo}

\author{
Raúl Vargas ${ }^{1 *}$, Andrés G. Marrugo ${ }^{1}$, Jesús Pineda ${ }^{1}$, Jaime Meneses ${ }^{3}$, Lenny A. Romero ${ }^{2}$ \\ 1. Facultad de Ingeniería, Universidad Tecnológica de Bolívar, Cartagena, Colombia \\ 2. Facultad de Ciencias Básicas, Universidad Tecnológica de Bolívar, Cartagena, Colombia \\ 3. Grupo de Óptica y Tratamiento de Señales, Universidad Industrial de Santander, Bucaramanga, Colombia \\ *E-mail: raulandres 16@hotmail.com
}

Received: 18/12/2017 Accepted: 03/09/2018

DOI: $10.7149 /$ OPA.51.3.50305

\begin{abstract}
:
The calibration methods most used in fringe projection profilometry are based on models of least squares adjustment and stereo vision techniques. However, the geometric distortions of the projector and camera lenses introduce imprecision in certain regions of the 3D reconstruction. In this paper, we perform a comparative study between the second order polynomial adjustment method and the stereo calibration method applying lens distortion compensation. The experimental results show that in the stereo calibration the incidence of the distortions in the 3D reconstruction is significant. In contrast, in the proposed polynomial calibration, reconstruction errors are associated with the calibrated volume, typically low within the calibration volume.
\end{abstract}

Key words: Fringe projection, stereo-vision, lens geometric distortions, polynomial calibration.

\section{RESUMEN:}

Los métodos de calibración más usados en perfilometría por proyección de franjas están basados en modelos de ajustes por mínimos cuadrados y técnicas de visión estéreo. Sin embargo, las distorsiones geométricas de los lentes del proyector y de la cámara introducen imprecisión en ciertas regiones de la reconstrucción 3D. En este trabajo realizamos un estudio comparativo entre el método de ajuste polinomial de segundo orden y el método de calibración estéreo aplicando compensación de distorsiones. Los resultados experimentales muestran que en la calibración estéreo la incidencia de las distorsiones en la reconstrucción 3D es significativa. En cambio, en la calibración polinomial propuesta, los errores de reconstrucción están asociados al volumen calibrado.

Palabras clave: Proyección de franjas, visión estéreo, distorsiones geométricas, calibración polinomial.

\section{REFERENCES AND LINKS / REFERENCIAS Y ENLACES}

[1] L. Felipe-Sesé, F. A. Díaz, and P. Siegmann, "Integration of Fringe Projection and 2D Digital Image Correlation for the measurement of 3D displacements and strains," Opt. Pura Apl., 50, pp. 25-35, (2017).

[2] S. S. Gorthi and P. Rastogi, "Fringe projection techniques: whither we are?," Opt. Lasers Eng., 48, pp. 133-140, (2010).

[3] A. Hanafi, T. Gharbi, and J.-Y. Cornu, "In vivo measurement of lower back deformations with Fourier- 
transform profilometry.," Appl. Opt,. 44, pp. 2266-2273, (2005).

[4] J. Barrios, M. Morón, C. Barrios, R. Contreras, A. González, and J. Meneses, “Three-dimensional scanning of the cornea by using a structured light module," Opt. Pura y Apl., 50, pp. 351-357, (2017).

[5] Q. Guo, Y. Ruan, J. Xi, L. Song, X. Zhu, Y. Yu, and J. Tong, "3D shape measurement of moving object with FFT-based spatial matching," Opt Laser Technol, 100, pp. 325-331, (2018).

[6] L. Huang, P. S. K. Chua, and A. Asundi, "Least-squares calibration method for fringe projection profilometry considering camera lens distortion.," Appl. Opt., 49, pp. 1539-1548, (2010).

[7] M. Takeda and K. Mutoh, "Fourier transform profilometry for the automatic measurement of 3-D object shapes.," Appl. Opt., 22, p. 3977, (1983).

[8] A.-S. Poulin-Girard, S. Thibault, and D. Laurendeau, "Influence of camera calibration conditions on the accuracy of 3D reconstruction," Opt Express, 24, p. 2678, (2016).

[9] Y. Wen, S. Li, H. Cheng, X. Su, and Q. Zhang, "Universal calculation formula and calibration method in Fourier transform profilometry.," Appl. Opt., 49, pp. 6563-6569, (2010).

[10] J. Lu, R. Mo, H. Sun, and Z. Chang, "Flexible calibration of phase-to-height conversion in fringe projection profilometry," Appl. Opt., 55, p. 6381, (2016).

[11] S. Zhang and P. S. Huang, "Novel method for structured light system calibration," Opt. Eng., 45, 083601, (2006).

[12] P. Stavroulakis, D. Sims-Waterhouse, S. Piano, and R. Leach, "Flexible decoupled camera and projector fringe projection system using inertial sensors," Opt. Eng. 56, no. 10, pp. 1-6, (2017).

[13] H. Liu, W.-H. Su, K. Reichard, and S. Yin, "Calibration-based phase-shifting projected fringe profilometry for accurate absolute 3D surface profile measurement," Optics Commun., 216, pp. 65-80, (2003).

[14] X. Liu, Z. Cai, Y. Yin, H. Jiang, D. He, W. He, Z. Zhang, and X. Peng, "Calibration of fringe projection profilometry using an inaccurate 2D reference target," Opt. Lasers Eng., 89, pp. 131-137, (2017).

[15] Y. Yin, X. Peng, A. Li, X. Liu, and B. Z. Gao, "Calibration of fringe projection profilometry with bundle adjustment strategy.," Opt. Lett, 37, pp. 542-544, (2012).

[16] D. Acevedo and J. Meneses, "Global positioning system of an object using high resolution stereo vision," Opt. Pura y Apl., 45, pp. 307-313 (2012).

[17] W. Zhang, W. Li, L. Yu, H. Luo, H. Zhao, and H. Xia, "Sub-pixel projector calibration method for fringe projection profilometry," Opt Express, 25, p. 19158, (2017).

[18] Z. Huang, J. Xi, Y. Yu, and Q. Guo, "Accurate projector calibration based on a new point-to-point mapping relationship between the camera and projector images," Appl. Opt., 54, pp. 347-356, (2015).

[19] S. Zhang, High-Speed 3D Imaging with Digital Fringe Projection Techniques. CRC Press, (2016).

[20] J.-Y. Bouguet, "Camera calibration toolbox for Matlab," http://www.vision.caltech.edu/bouguetj/calib doc/index.html , (2004)

[21] Z. Zhang, "A flexible new technique for camera calibration," IEEE Trans. Pattern Anal. Mach. Intell., 22, pp. 1330-1334, (2000).

\section{Introduction}

Three-dimensional (3D) scanning techniques based on fringe pattern projection and imaging have been extensively developed to meet the demands of many applications including, industrial inspection, computer-aided design, manufacturing, medical diagnosis, etc., [1]-[4]. The main advantages of these techniques include their non-contact nature, dense measurement, high speed, high automation degree and high accuracy [5], [6]. Fringe projection profilometry (FPP) consists in projecting a sinusoidal pattern onto an object to measure its shape and obtain a 3D digital model. The process is based on phase retrieval techniques from the projected patterns and a mapping from the recovered phase to the physical measurement values. This unit conversion, or phase-to-height mapping, depends on the geometric parameters of the FPP system (rotation angles and translation vectors between the projection and capture devices). The calibration of the FPP system is a crucial step for achieving accurate measurements. Many calibration methods have been proposed, however they can be broadly classified in two categories: phaseto-height mapping methods [7]-[10] or stereo-vision methods [2], [11], [12]. In the first category, the relations between phase and the height of the object have to be identified and constructed either by a modelbased approach or a polynomial fit [6], [13]. These techniques often work by displacing a plane, 
perpendicular to the camera axis, in accurately known values and establishing the phase-to-height relation for each pixel in the camera. In the second category, the camera and the projector form a calibrated stereo vision system, in which the projector is regarded as an inverse camera [11], [12], [14]-[16]. However, as opposed to a traditional stereo vision system, the fringe projection approach does not require feature matching. The camera and projector pixel correspondences are obtained directly from the recovered phase, thus enabling the triangulation for estimating the 3D coordinates of object's surface. It is also worth noting that this approach is faster and more flexible in that accurate displacements are not required [11], [17], [18].

In the majority of calibration approaches, the authors assume a linear camera model, i.e. one without lens distortions [11]. This is often done on the assumption that the radial and tangential distortion coefficients are negligible or that the accuracy of the FPP system is sufficient under a camera model. However, the influence of the lens distortions (both camera and projector lenses) in different FPP calibration methods has not been sufficiently studied. Moreover, most works reported in the literature limit the error analysis to reprojection error, but the ultimate test is to determine the influence on 3D reconstruction error [17].

In this work, we carry out a comparative study from a second-degree polynomial and stereo calibration methods. In addition, we study the influence of lens distortions in the 3D reconstruction by reconstructing the same object under the different calibration approaches. In the following sections we describe each calibration method with the calibration results on our experimental setup. Then we compare both methods by carrying out the 3D reconstruction of a flat board from different orientations. Also, two validation procedures were carried out to validate the calibration methods. Finally, we discuss the overall performance and tradeoffs for selecting the best calibration approach for a given application.

\section{Experimental Setup}

In Fig. 1 we show the experimental setup, which consists of a monochromatic CMOS camera (Basler Ace$1300 \mathrm{gm}$; $1282 \times 1026$-60 fps) with lens Edmund Optics-58001 with focal length of $12 \mathrm{~mm}$ at F1.8, a DLP® projector (DELL M115HD; 1280x800 with projection distance of 0.97-2.58 m.), a linear translation stage with $0.04 \mathrm{~mm}$ precision controlled from a computer, a checkerboard for calibration with $10 \times 10 \mathrm{~mm}$ squares and a PC workstation. To synchronize the camera and DLP projector, we duplicate the VGA signal from the computer using a VGA splitter and connecting the vertical sync pulse to the hardware trigger pin of the camera [19].

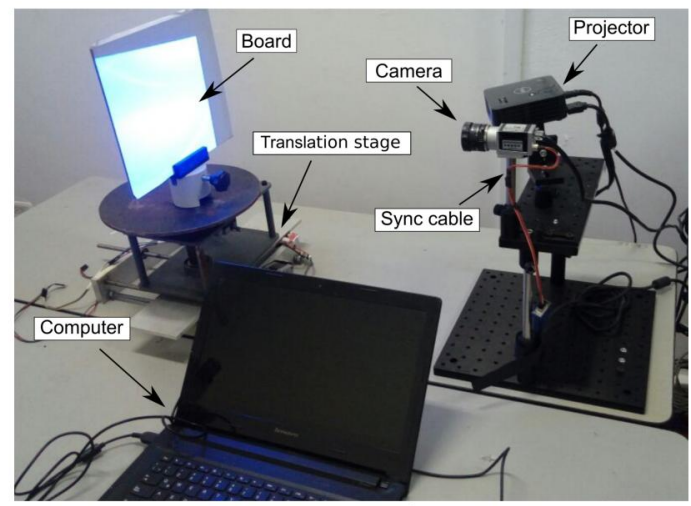

Fig 1. Experimental System.

\section{Stereo Calibration}

In this work, we use two stereo calibration methods based on a linear projection model (LPM) proposed by Zhang and Huang [11], and the same method with the lens distortion model added (LDM). The cameraprojector is considered as a binocular framework and by searching for homologous image points in the camera and projector. The 3D coordinates of the measured points can be reconstructed once system parameters have been determined. To compute the system parameters and search corresponding points, horizontal and vertical fringes are projected onto a calibration plane placed in different positions and orientations. In our case, we used 15 different positions. This plane is a checkerboard target with two colors, 
which are selected so that with gray-level projection the monochromatic camera response to the two colors is similar. However, when the target is illuminated with red light, only the checkerboard image is detected in the camera. For each calibration plane position, we capture the images shown in Fig. 2. Using the vertical (Fig. 2(b)) and horizontal fringe images (Fig. 2(d)), we extract the discontinuous phases by Fourier transform profilometry [7]. Afterward, we apply a phase unwrapping algorithm using a centerline image (Fig. 2(c) and Fig. 2(e)) to obtain the absolute continuous phase maps in the horizontal and vertical directions.

In Fig. 3, we show a schematic of a fringe projection stereo system. In this setup, the projector is considered as a camera and the calibration relies on obtaining a relation between the projected image plane $\left(u^{p}, v^{p}\right)$ and the captured image plane $\left(u^{c}, v^{c}\right)$. Considering a point $\left(x^{m}, y^{m}, z^{m}\right)$ in the checkerboard coordinate system, we find its corresponding coordinate in the camera and projector systems using the following equations, respectively,

$$
\begin{gathered}
s_{c}\left[\begin{array}{c}
u^{c} \\
v^{c} \\
1
\end{array}\right]=\left[\begin{array}{ccc}
f_{u}^{c} & 0 & C_{u}^{c} \\
0 & f_{v}^{c} & C_{v}^{c} \\
0 & 0 & 1
\end{array}\right]\left[^{c} R_{m},{ }^{c} T_{m}\right]\left[\begin{array}{c}
x^{m} \\
y^{m} \\
z^{m} \\
1
\end{array}\right], \\
s_{p}\left[\begin{array}{c}
u^{p} \\
v^{p} \\
1
\end{array}\right]=\left[\begin{array}{ccc}
f_{u}^{p} & 0 & C_{u}^{p} \\
0 & f_{v}^{p} & C_{v}^{p} \\
0 & 0 & 1
\end{array}\right]\left[{ }^{p} R_{m},{ }^{p} T_{m}\right]\left[\begin{array}{c}
x^{m} \\
y^{m} \\
z^{m} \\
1
\end{array}\right],
\end{gathered}
$$

where $s_{c}$ and $s_{p}$ are scaling factors; $f_{u} \quad f_{v} C_{u}$ and $C_{v}$ are intrinsic parameters of the camera and the projector pinhole model; $\left[{ }^{c} R_{m},{ }^{c} T_{m}\right]$ y $\left[{ }^{p} R_{m},{ }^{p} T_{m}\right]$ are transformation matrices from the coordinate system of the model $\left(X^{m}, Y^{m}, Z^{m}\right)$ and the camera and projector systems, respectively.

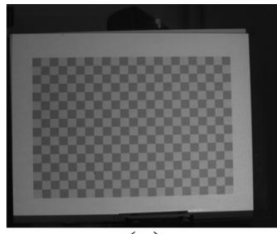

(a)

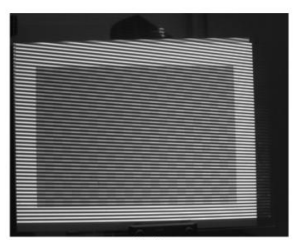

(b)

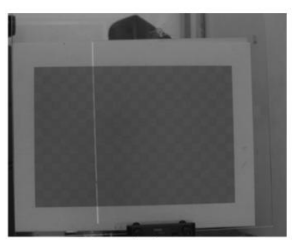

(c)

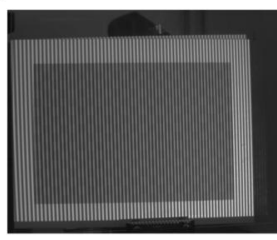

(d)

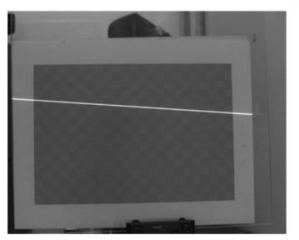

(e)

Fig 2. Images captured in each position of calibration plane projecting (a) Red light, (b) Vertical fringes, (c) Vertical center line, (d) Horizontal fringes and (e) Horizontal center line. 


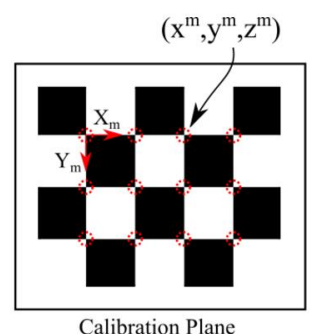

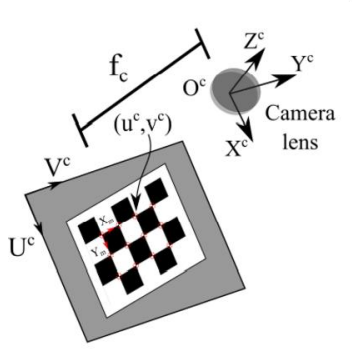

Camera image plane

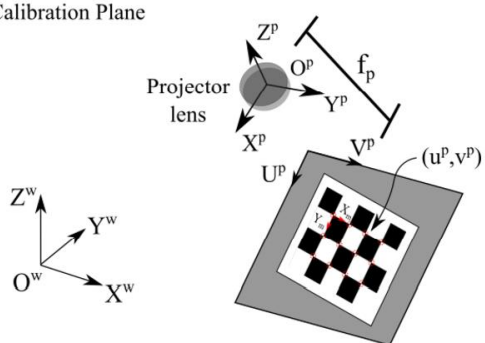

Projector image plane

Fig. 3. Stereo model of camera-projector system

In this paper, we consider the camera and projector distortion as [8],

$$
\left[\begin{array}{l}
u_{d} \\
v_{d}
\end{array}\right]=\left(1+k_{1} r_{n}^{2}+k_{2} r_{n}^{4}+k_{3} r_{n}^{6}\right)\left[\begin{array}{l}
u_{n} \\
v_{n}
\end{array}\right]+\left[\begin{array}{l}
2 p_{1} u_{n} v_{n}+p_{2}\left(r_{n}^{2}+2 u_{n}^{2}\right) \\
2 p_{2} u_{n} v_{n}+p_{1}\left(r_{n}^{2}+2 v_{n}^{2}\right)
\end{array}\right],
$$

where, $r_{n}^{2}=u_{n}^{2}+v_{n}^{2} ; k_{1}, k_{2}$ and $k_{3}$ are coefficients of radial distortion; $p_{1}$ and $p_{2}$ are coefficients of tangential distortion; $\left[u_{d}, v_{d}\right]^{t}$ are the normalized coordinates of the pinhole with distortion and $\left[u_{n}, v_{n}\right]^{t}$ the new normalized coordinate with distortion compensated.

\section{3.a. Camera-projector Correspondence}

To find the stereo parameters of the system, we must relate common points in the camera and projection systems. In addition, these points must have a known position in the world or model coordinate system, in our case, the checkerboard pattern. The vertical and horizontal fringe patterns are used to establish a relationship between the camera image plane and the projector image plane. Recall, that we consider the projector as a camera. Based on the Fourier transform [7] we can extract the wrapped phase of the captured fringe images. Then, we apply a phase unwrapping algorithm to obtain the continuous phase. By using the central projection lines, we obtain the absolute phase maps in horizontal and vertical directions. These phase maps are related to the projector coordinates by,

$$
\begin{aligned}
u^{p} & =N_{u} \frac{\Phi_{v}}{2 \pi}+\frac{w}{2} \\
v^{p} & =N_{v} \frac{\Phi_{h}}{2 \pi}+\frac{h}{2}
\end{aligned}
$$

where, $\Phi_{u}$ and $N_{u}$ are the absolute phase map and period of the vertical fringe, respectively; $\Phi_{h}$ and $N_{v}$ are the absolute phase map and the period of the horizontal fringes, respectively; $w$ and $h$ are the width and height of the fringe patterns. We detect the corners of the checkered board pattern in the camera image with sub-pixel precision using standard feature detection techniques [20]. The phase values in the corners can be calculated fitting the phase maps $\Phi_{v}$ and $\Phi_{h}$ to a third-degree polynomial surface and evaluating it at the corner coordinates. It should be noted that in the original work by Zhang and Huang [11], the authors synthesize an artificial projector image as if captured by the projector to obtain its calibration parameters. However, this is not necessary and leads to inaccuracy. In this work, we propose to only transform the subpixel coordinates for obtaining the projector parameters. 


\section{3.b. Stereo 3D reconstruction}

In order to obtain the 3D reconstruction from the stereo system, we must determine the correspondence between an observed point on the camera and the projector plane. From equations (1) and (2), we need to solve three non-linear equations $f\left(\right.$.) to obtain the $3 \mathrm{D}$ coordinates of a given point $\left(x^{w}, y^{w}, z^{w}\right)$. The reconstruction equations are

$$
\begin{aligned}
& f_{1}\left(x^{w}, y^{w}, z^{w}, u^{c}\right)=0, \\
& f_{2}\left(x^{w}, y^{w}, z^{w}, v^{c}\right)=0, \\
& f_{3}\left(x^{w}, y^{w}, z^{w}, u^{p}\right)=0,
\end{aligned}
$$

where $u^{c}, v^{c}$, and $u^{p}$ are known. Therefore, the world coordinates $\left(x^{w}, y^{w}, z^{w}\right)$ of a point can be uniquely solved for the image point $\left(u^{c}, v^{c}\right)$.

\section{3.c. Stereo Calibration Results}

We used the method by Zhang [21] and the Bouget camera calibration toolbox [20] for obtaining the intrinsic and extrinsic parameters of the stereo system with and without the distortion model included. The parameters obtained for LPM and LDM are registered in Table 1 and Table 2, respectively. The principal point of the projector in the v-direction, in both models, is closer to the row numbers of the projector. Its values are 793.09 and 787.15, where the number of pixels in that direction is 800 . This is expected as these devices are manufactured for projecting with a vertical offset from the optical axis. In Fig. 4 we show the reprojection errors of the camera and projector for both considered models. The mean reprojection errors using an LPM for the camera and projector were 0.472 and 0.160 pixels, respectively. However, these errors are reduced to 0.164 and 0.135 pixels when the LDM is added to LPM.

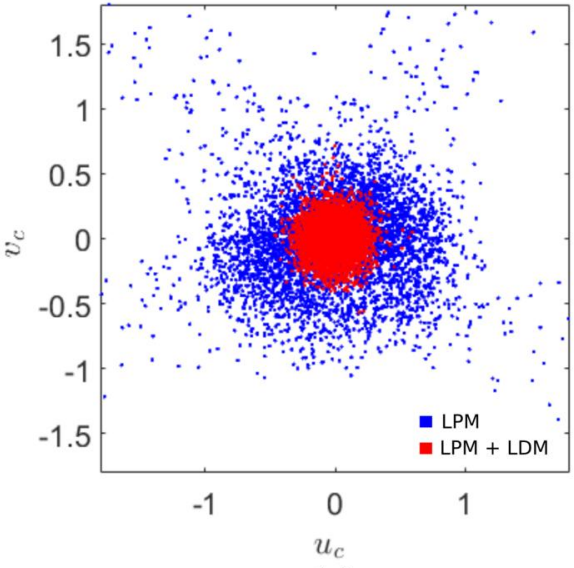

(a)

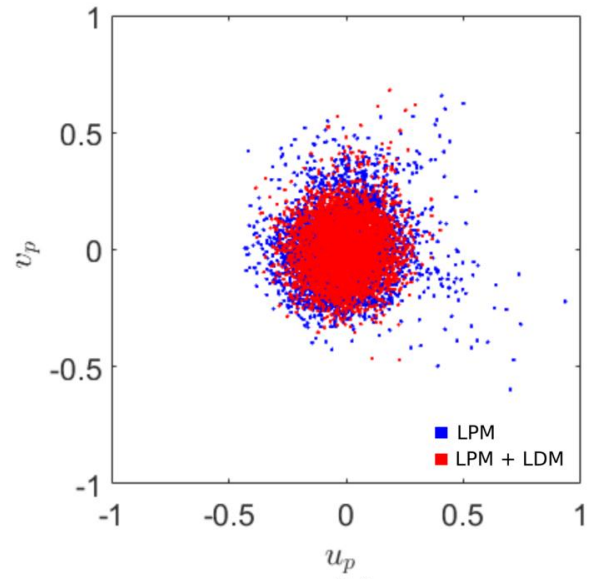

(b)

\begin{tabular}{|c|c|c|c|c|c|}
\hline Parameters & Camera & \multicolumn{4}{|c|}{ Projector } \\
\hline$f_{u}$ & $2314.71 \pm 9.55$ & \multicolumn{4}{|c|}{$1946.06 \pm 3.52$} \\
\hline$f_{v}$ & $2320.92 \pm 5.58$ & \multicolumn{4}{|c|}{$1949.37 \pm 3.66$} \\
\hline$C_{u}$ & $597.49 \pm 2.95$ & \multicolumn{4}{|c|}{$643.15 \pm 1.84$} \\
\hline$C_{v}$ & $524.73 \pm 3.22$ & \multicolumn{4}{|c|}{$793.09 \pm 1.10$} \\
\hline & $\begin{array}{lll:l}1 & 0 & 0 & 0\end{array}$ & 0.9162 & -0.0159 & 0.4004 & -186.726 \\
\hline & $\begin{array}{lll:l}0 & 1 & 0 & 0\end{array}$ & 0.0089 & 0.9998 & 0.0195 & -117.120 \\
\hline$\left[{ }^{\mathrm{w}} \mathrm{R} \mid{ }^{\mathrm{w}} \mathrm{t}\right]$ & $\begin{array}{lll:l}0 & 0 & 1 & 0\end{array}$ & -0.4006 & -0.0014 & 0.9161 & 96.972 \\
\hline
\end{tabular}

Fig. 4. Reprojection errors in pixels of pinhole model proposed for (a) camera and (b) projector with LPM and LDM added.

Table 1. Camera and projector calibration results using LPM. 
Table 2. Camera and projector calibration results with LDM added.

\begin{tabular}{|c|c|c|c|c|}
\hline Parameters & Camera & \multicolumn{3}{|c|}{ Projector } \\
\hline$f_{u}$ & $2277.21 \pm 3.28$ & \multicolumn{3}{|c|}{$1936.11 \pm 3.11$} \\
\hline$f_{v}$ & $2279.43 \pm 3.30$ & \multicolumn{3}{|c|}{$1935.47 \pm 3.24$} \\
\hline$C_{u}$ & $612.21 \pm 2.81$ & \multicolumn{3}{|c|}{$606.51 \pm 3.28$} \\
\hline$C_{v}$ & $482.36 \pm 2.02$ & \multicolumn{3}{|c|}{$787.15 \pm 2.09$} \\
\hline$k_{1}$ & $-0.218 \pm 0.015$ & \multicolumn{3}{|c|}{$0.006 \pm 0.007$} \\
\hline$k_{2}$ & $0.319 \pm 0.522$ & \multicolumn{3}{|c|}{$-0.251 \pm 0.087$} \\
\hline$k_{3}$ & $-0.021 \pm 5.107$ & \multicolumn{3}{|c|}{$0.365 \pm 0.378$} \\
\hline$p_{1}$ & $-0.0015 \pm 0.0002$ & \multicolumn{3}{|c|}{$-0.004 \pm 0.001$} \\
\hline$p_{2}$ & $-0.0015 \pm 0.0002$ & \multicolumn{3}{|c|}{$-0.005 \pm 0.001$} \\
\hline & $\begin{array}{lll:l}1 & 0 & 0 & 0\end{array}$ & 0.9644 & $-0.0106 \quad 0.4266$ & -181.059 \\
\hline & $\begin{array}{lll:l}0 & 1 & 0 & 0\end{array}$ & 0.0092 & $0.9999 \quad 0.0052$ & -117.518 \\
\hline$\left[\left.{ }^{\mathrm{w}} \mathrm{R}\right|^{\mathrm{w}} \mathrm{t}\right]$ & $\begin{array}{lll:l}0 & 0 & 1 & 0\end{array}$ & -0.4266 & $-0.0008 \quad 0.9044$ & 110.677 \\
\hline
\end{tabular}

\section{Polynomial Calibration}

The second calibration method that we implemented is a direct phase-to-height approach. Most phase-toheight methods depend on the system geometric parameters, such as angles and distances between the camera and the projector. However, often these parameters are difficult to determine precisely. Therefore, several techniques have been proposed to calibrate the system indirectly using a phase-depth mapping via a least-squares fit of the phase to height. Our polynomial calibration method consists in displacing a reference plane in known $Z_{n}$ distances, as shown in Fig. 5. In each displacement, the phase difference is calculated as,

$$
\Delta \varphi_{n}=\varphi_{n}-\varphi_{0},
$$

where $\phi_{n}$ is the absolute phase map in each position plane and $\phi_{0}$ is the absolute phase of the reference plane. In this paper, we use a second-degree polynomial calibration as,

$$
Z=a_{z} \Delta \varphi^{2}+b_{z} \Delta \varphi+c_{z}
$$

where $\mathrm{Z}$ is the height of a surface measured from the reference plane and $\Delta \phi$ is the phase difference between the reconstructed object and the reference plane. Z-calibration is known as out-of-plane calibration. Once $\mathrm{Z}$ is calibrated, an analogous procedure is performed to convert the pixel position in the image sensor matrix to millimeters coordinates along $\mathrm{X}$ and $\mathrm{Y}$ directions. The polynomial coefficients are calculated for each pixel on the image using the least squares method. 


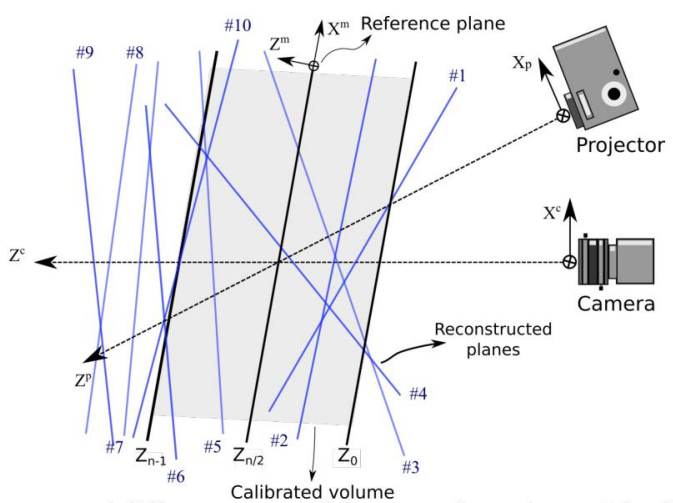

Fig. 5. Schematic of the triangulation setup and different poses of the target for polynomial calibration and planes reconstructed for distortion analysis.

\section{4.a. Polynomial Calibration Results}

The calibration plane was placed slightly rotated with respect to the $\mathrm{Z}$ axis of the camera. The plane was displaced in the $\mathrm{Z}$ direction of the model (perpendicular to the plane) with $1.6 \mathrm{~mm}$ increments from $Z_{0}=0 \mathrm{~mm}$ to $Z_{59}=94.4 \mathrm{~mm}$, for a total of 60 positions. For each displaced position, three Images as the ones shown in Fig. 2(a)-(c) were captured. The absolute continuous phase of each plane was extracted by Fourier transform profilometry using the fringe pattern and the projected centerline. The checkerboard is used to relate the metric corners coordinates with their phase values, in their respective subpixel position on the image. The reference plane chosen was $Z_{30}$ and the calibrated volume was $210 \mathrm{~mm} \times 140 \mathrm{~mm} \times 94.4 \mathrm{~mm}$ ( $\mathrm{X}, \mathrm{Y}$, and $\mathrm{Z}$ directions, respectively) with a fit RMS error for each pixel, along the 60 positions, shown in Fig. 6. We observe how the error varies across the field of view with relatively high values near the border of the captured image. This is probably due to a slight rotation between the plane and the displacement direction. To compensate the lens distortions it is necessary to use the camera parameters found in stereo calibration with LDM and to rectify the acquired images before processing them.

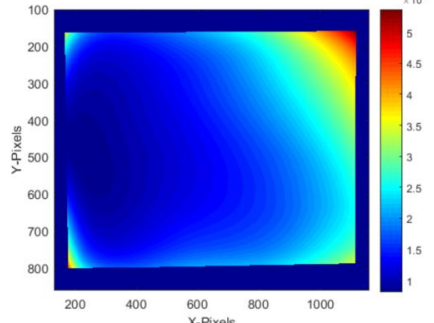

(a)

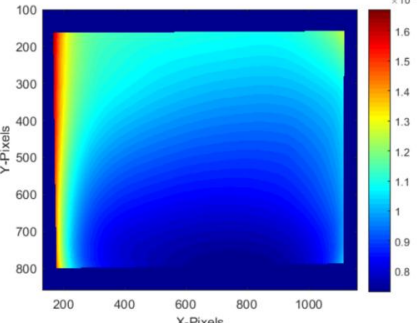

(b)

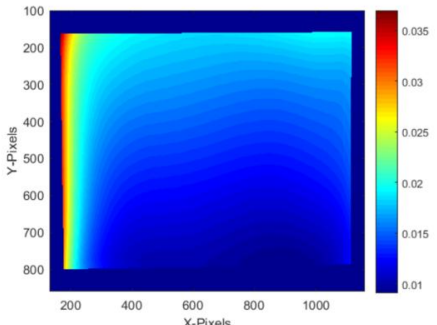

(c)

Fig. 6. RMS error maps of (a) X-calibration, (b) Y-calibration and (c) Z-calibration.

\section{Experiments}

\section{6.a 3D Reconstruction and Distortion Analysis}

To study the accuracy of each method and measure the influence of lens distortions, we reconstructed 10 planes placed in different positions and rotations as shown in Fig. 5. The planes were reconstructed by the stereo and polynomial calibration methods, with and without distortion compensation. For each reconstructed plane, an ideal plane was adjusted using least squares and an RMS error was obtained from the subtraction of the planes. The results are shown in Table 3.

For the stereo calibration approach, a significant reduction of the RMS error is observed for the ten planes when we compensate for the distortions, the error reduction for all of the planes corresponds to a decrease in more than $65 \%$. In general, the 10 planes are reconstructed with a similar precision with respect to different positions and orientations. However, for this calibration the correction of the distortions is ultimately necessary. 
In the polynomial calibration, we noticed that there are planes that are reconstructed with a lower error than others. This is because several of these planes are mainly inside the calibration volume (\# 2, \# 4 and \#5) and others are totally or mainly outside (\#6, \#9,\#10). Then, we find the RMS errors of only the region of the planes within calibrated volume. Subsequently, results are shown in Table 4. We observe that these regions are reconstructed with high precision and the error is similar for all the planes. When compensating the distortions in these regions we notice a slight reduction of the error in some planes, but that is not as significant as in the stereo calibration.

Table 3. RMS errors in millimeters of ten planes reconstructed with polynomial and stereo calibration. No: without lens distortion

\begin{tabular}{|c|c|c|c|c|}
\multirow{2}{*}{} & \multicolumn{2}{c}{ compensation, Yes: with lens distortion compensation. } \\
\cline { 2 - 5 } & \multicolumn{2}{c|}{ Stereo calibration } & \multicolumn{2}{c|}{ Polynomial calibration } \\
\hline \hline Plane & No & Yes & No & Yes \\
\hline \hline 1 & 0.7090 & 0.2275 & 0.1377 & 0.1389 \\
3 & 0.7167 & 0.2293 & 0.0835 & 0.0815 \\
4 & 0.7648 & 0.2089 & 0.1740 & 0.1600 \\
5 & 0.7571 & 0.1578 & 0.1010 & 0.0866 \\
6 & 0.6459 & 0.1651 & 0.1363 & 0.1315 \\
7 & 0.5760 & 0.1574 & 0.3159 & 0.3137 \\
8 & 0.5745 & 0.1281 & 0.1207 & 0.1180 \\
9 & 0.5028 & 0.1561 & 0.0997 & 0.0927 \\
10 & 0.6965 & 0.1720 & 0.4488 & 0.4834 \\
\hline \hline
\end{tabular}

Table 4. RMS errors in millimeters inside polynomial calibration volume of ten planes reconstructed. No: without lens distortion compensation, Yes: with lens distortion compensation. Incidence: percentage of plane area that is within the calibrated volume. ${ }^{*}$ )

\begin{tabular}{|c|c|c|c|}
\hline \multicolumn{4}{|c|}{ These planes are completely outside the calibration volume. } \\
\hline \hline Plane & No & Yes & Incidence (\%) \\
\hline 1 & 0.0468 & 0.0423 & 58.73 \\
3 & 0.0504 & 0.0463 & 59.07 \\
4 & 0.0612 & 0.0588 & 64.43 \\
5 & 0.0586 & 0.0588 & 63.72 \\
6 & 0.0686 & 0.0588 & 48.00 \\
$7^{*}$ & 0.0563 & 0.0565 & 3.55 \\
$8^{*}$ & - & - & 0.00 \\
$9 *$ & - & - & 0.00 \\
10 & - & - & 0.00 \\
\hline
\end{tabular}

\section{6.b Validation}

We carried out two experiments to validate the polynomial and stereo calibration models with LDM. In the first procedure, a plane was displaced using a translation unit with a micrometric screw with a resolution of $0.001 \mathrm{~mm}$. Ten consistent displacements of $0.100 \pm 0.001 \mathrm{~mm}$ were executed in perpendicular orientation to the displaced plane. The displaced planes were reconstructed and adjusted by least squares to an ideal 
plane and we calculated the distances between them. For the polynomial calibration, we found an average displacement with standard deviation of $0.097 \pm 0.012 \mathrm{~mm}$, while for the stereo calibration an average displacement of $0.094 \pm 0.007 \mathrm{~mm}$ was found, these measurements correspond to relative errors of $2.6 \%$ and $6 \%$, respectively.s

In the second validation experiment, we used an aluminum object shown in Fig. 6a. The object was machined by a CNC (Computer Numerical Control) milling machine with a tolerance of $0.001 \mathrm{~mm}$. We painted the object surface in white for improving the measurement conditions. Using the polynomial technique, we reconstructed the object obtaining the depth map shown in Fig. 6b. This figure shows the presence of three surface steps (R0, R1, and R2), where the reference distances between the steps R0-R1 and R0-R2 are $1.500 \pm 0.001 \mathrm{~mm}$ and $3.000 \pm 0.001 \mathrm{~mm}$, respectively. From the reconstructed surface, we calculated the average distances with its standard deviation of R0-R1 and R0-R2, were $1.505 \pm 0.061 \mathrm{~mm}$ and $2.988 \pm 0.065 \mathrm{~mm}$, respectively. These measurements correspond to a relative error of $0.33 \%$ and $0.40 \%$. Fig. $6 \mathrm{c}$ shows the points associated with the red profile shown in Fig. $6 \mathrm{~b}$ and the real references of the steps of the object.

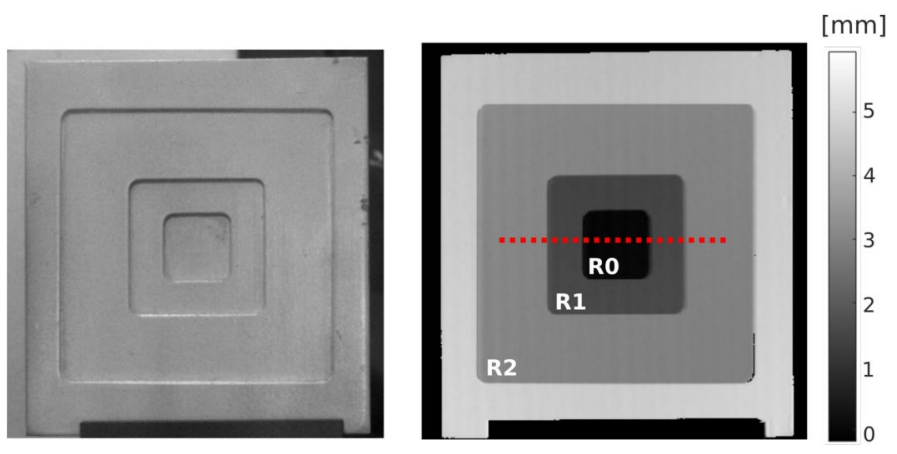

(a)

(b)

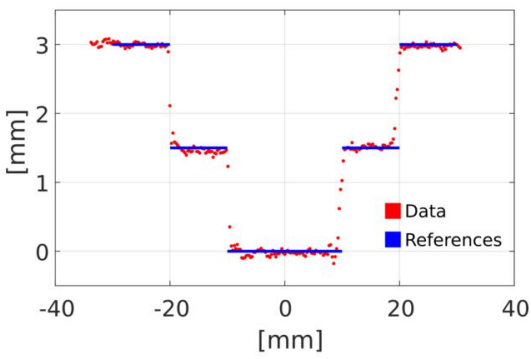

(c)

Fig. 7. (a) Validation pattern object. (b) Depth map of the reconstructed object. (c) profile of a section of the reconstructed object.

\section{Conclusions}

In this work, we compared a polynomial and a stereo calibration method, and in particular we studied the influence of the geometric distortion compensation of the camera and projector lenses in each method. The experimental results show that the compensation of the lens distortions in the stereo calibration approach has an important effect on the reconstruction precision with a decrease of RMS reconstruction error of over $50 \%$ in most cases. This is expected since the triangulation is based on a linear model of the camera and the projector. In contrast, the compensation of geometric distortions has a minor effect on the polynomial calibration. Nevertheless, we showed that if the object is placed within the calibration volume compensating the distortions does decrease the reconstruction error.

The polynomial calibration offers greater precision in reconstruction than the stereo calibration at the expense of a much more elaborate procedure. Its implementation requires a precise displacement system, and in certain cases, a precise alignment of the optical systems. Furthermore, in order to maintain high precision, the calibration volume limits the location of the objects for reconstruction. Conversely, the stereo calibration has a faster and more versatile implementation, because precise displacements are not required and there is no restriction regarding a calibrated volume. Although in general, stereo systems do have a depth dependent uncertainty.

\section{Acknowledgements}

This work has been partly funded by Colciencias (Fondo Nacional de Financiamiento para la Ciencia, la Tecnología y la Innovación "Francisco José de Caldas") project 538871552485, and by Universidad Tecnológica de Bolívar project FI1607T2001. R. Vargas and J. Pineda thank the Universidad Tecnológica de Bolívar for a Master's degree scholarship. 